\title{
Degenerative spondylolisthesis does not influence surgical results of laminoplasty in elderly cervical spondylotic myelopathy patients
}

\author{
Hideki Shigematsu
}

Received: 9 March 2009/Revised: 5 February 2010/Accepted: 11 February 2010/Published online: 27 February 2010

(C) Springer-Verlag 2010

\begin{abstract}
The objective of the study was to investigate the comorbidity of degenerative spondylolisthesis (DS), in elderly cervical spondylotic myelopathy (CSM) patients in our hospital, and the correlation between surgical results and preoperative DS. There are few studies on the outcome of laminoplasty for CSM with DS. A total of 49 elderly patients ( $>65$ years old) who eventually had surgical treatment for CSM were evaluated. A slippage displacement of more than $2.5 \mathrm{~mm}$ at least at one level was classified to have a positive DS on flexion/extension radiographs (DS group). A slippage displacement less than $1.0 \mathrm{~mm}$ was considered a negative DS (non-DS group). Seventeen patients who had slippage of $1.0-2.5 \mathrm{~mm}$ were excluded from the study. The DS group $(n=15)$ included cases with DS at preoperation, while the remaining cases ( $n=17$ ) belonged to the non-DS group. The flexion/ extension radiographs of the two groups were compared for range of motion and clinical results at 3 years after the operation. Of all elderly patients, $30.6 \%$ had DS. There was no significant difference between the two groups based on the clinical results. The range of motion of all cervical spines (DS group and non-DS group) was significantly limited. However, there was no significant difference between the two groups. New postoperative DS appeared in four patients, of which two were from the DS group and two from the non-DS group. These data suggest that degenerative spondylolisthesis does not influence surgical results in elderly cervical spondylotic myelopathy patients.
\end{abstract}

Keywords Cervical spondylotic myelopathy .

Spondylolisthesis · Degenerative instability $\cdot$ Laminoplasty

H. Shigematsu ( $\square)$

Nara Medical University, 840 Kashihara, Nara 634-8522, Japan

e-mail: shideki@naramed-u.ac.jp

\section{Introduction}

Cervical spondylotic myelopathy (CSM) may result from spinal cord compression and/or disturbed blood supply and is caused by degenerative changes or the instability of the cervical spine. In elderly Japanese CSM patients, the rate of occurrence of dynamic factors, such as instability of the vertebral column [1, 6-8, 29], was shown to be higher than for static factors, such as developmental canal stenosis [24], by radiological analysis [26]. Furthermore, CSM patients 65 years and older with spondylolisthesis had a high incidence of degenerative spondylolisthesis (DS) at C3-C4 or C4-C5 [14]. Thus, DS as an intervertebral instability factor may play an important causative role in CSM symptoms in elderly CSM patients. However, spondylolisthesis of the cervical spine has been considered rare in both the young $[3,5,17]$ and in the elderly [2, 4], and there are only a few studies focusing on the incidence of DS or surgical results of CSM with DS.

The aim of the present study was to investigate the comorbidity from DS among elderly CSM patients who underwent bilateral open-door laminoplasty in our hospital. Furthermore, we compared the surgical results of bilateral open-door laminoplasty between CSM patients with or without DS to determine whether DS, as an instability factor of the cervical spine, influenced surgical results.

\section{Materials and methods}

From 1990 to 2004, 194 patients with CSM (excluding myelopathy caused by ossification of the posterior longitudinal ligament, tumor, infection, trauma or athetoid cerebral palsy) were treated with laminoplasty in our department. Our operative indications were as follows. (1) 
One level of cord compression without developmental canal stenosis: anterior decompression and fusion. (2) One level of cord compression with developmental canal stenosis: laminoplasty. (3) More than two levels of cord compression: laminoplasty. Based on these indications, we performed laminoplasty without instrumentation, even if there was instability during the study period. Among these patients, 86 patients were more than 65 years old. We also excluded patients who had a previous history of anterior cervical fusion, or whose follow-up periods were less than 3 years. A total of 49 patients were included in this series.

Laminoplasty was performed by the double-door (midsagittal spinous-splitting) technique. Bilateral gutters were made at the $\mathrm{C} 3-\mathrm{C} 7$ laminae with a burr. After removing the spinous processes, the center of the lamina was split with a diamond burr and the lamina opened symmetrically (Fig. 1). A dome-shaped laminoplasty was performed for the C2 lamina [18]. Spinal instrumentation was not used for any surgery. Patients were confined to bed rest for 1-7 days, after which they were allowed to sit upright using a cervical collar. The cervical collars were recommended to be used for 1-2 months.

Clinical results were evaluated using Japanese Orthopedic Association scores (JOA scores; total points $=17$ ) at preoperation and at 3 years after operation [12], and the recovery rate was calculated according to the following formula: (JOA score at follow-up - preoperative JOA score $) /(17$ - preoperative JOA score $) \times 100(\%)$ [11].

Radiological evaluation was performed by plain and flexion/extension radiographs. Radiographs were taken at a 1.5-m film focus distance for each patient. DS as an
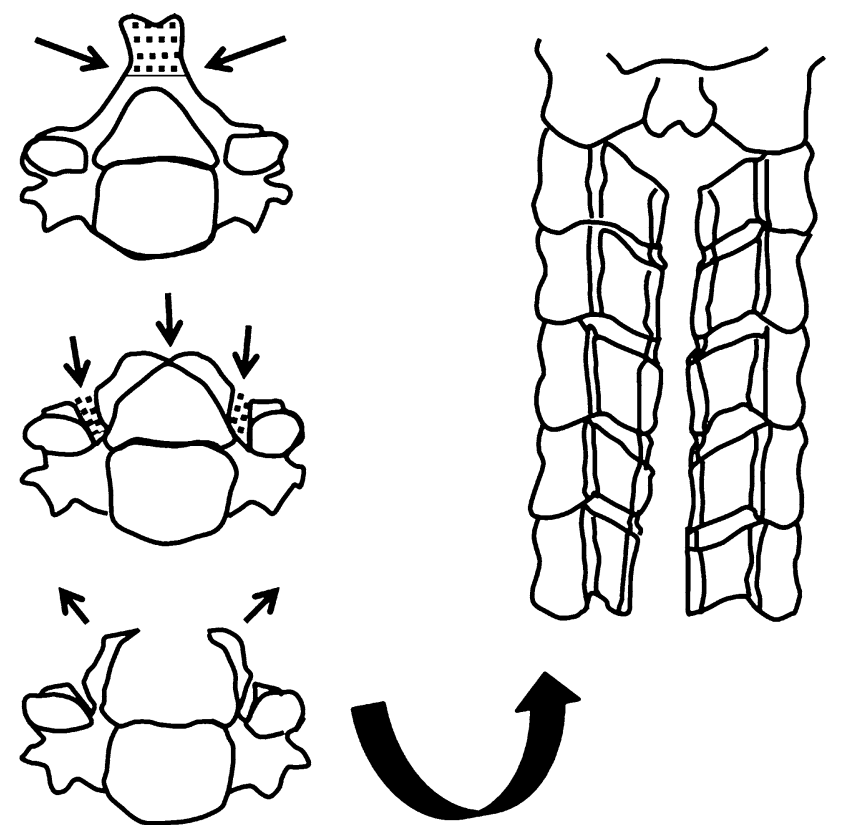

Fig. 1 Laminoplasty technique instability factor was considered to be present if more than $2.5 \mathrm{~mm}$ of slippage displacement was observed on flexion/ extension radiographs at least at one level (DS group; Fig. 2). For example, a 2-mm anterolisthesis at the flexion radiograph and a $1-\mathrm{mm}$ posterolisthesis at the extension radiograph were considered as $3 \mathrm{~mm}$ slippage displacement, which was classified in the DS group. On the other hand, less than $1.0 \mathrm{~mm}$ of slippage observed on flexion/ extension radiographs was classified as non-DS (non-DS group). Note that the C6-C7 was excluded as we could not evaluate slippage displacement at $\mathrm{C} 6-\mathrm{C} 7$ in some cases. We could not measure the instability below C6 because of the patients' shoulders. The 17 patients who had slippage of 1.0-2.5 mm were also excluded so as to compare strictly between the DS and the non-DS group. The range of motion of the cervical spine was measured according to Penning's method [21] based on the C2-C6 or C2-C7 angle in the flexion/extension lateral radiographs (Fig. 3). On plain radiographs taken with the patient in the neutral position, the sagittal cervical alignment was measured from C2 to C6 or C2 to C7 using Penning's method. Angles greater than $10^{\circ}, 0-10^{\circ}$, and $<0^{\circ}$ were judged to be lordotic, straight and kyphotic, respectively [13].

Statistical differences between the DS and non-DS groups were determined by $\chi^{2}$ analysis and Student's $t$ test. Differences with $p<0.05$ were considered to be significant.

\section{Results}

\section{Clinical outcomes}

The preoperative JOA score of all patients (DS and non-DS groups) was $6.7 \pm 3.6$ (mean $\pm \mathrm{SD}$ ). The JOA score at the final follow-up was $11.4 \pm 3.0(p<0.01)$ and the recovery rate was $46.6 \pm 20.1 \%$. According to our classification of preoperative spondylolisthesis as an instability factor, the DS group and the non-DS group comprised 15 and 17 patients, respectively (Table 1). The DS group included nine males and six females (mean age at surgery $=$ $72.4 \pm 6.3$ years). The non-DS group included 14 males and three females (mean age at surgery $=72.8 \pm 6.6$ years). There was no difference in the average age between the two groups $(p=0.87)$. There was no difference in the mean duration of symptoms between the DS group and the non-DS group (24.1 \pm 31.6 vs. $29.3 \pm 76.3$ months, respectively; $p=0.80)$ or in the mean preoperative JOA score between the DS group and the non-DS group (6.5 \pm 3.4 vs. $6.8 \pm 3.8$, respectively; $p=0.82$ ). There was no difference in the JOA score at 3 years after operation between the DS group and the non-DS group $(10.9 \pm 3.3$ vs. $11.9 \pm 2.8$, respectively; $p=0.35$ ) or in the mean recovery rates 
Fig. 2 Measurement of spondylolisthesis using functional radiographs. Slippage displacement $=\mathrm{a}+\mathrm{b}$

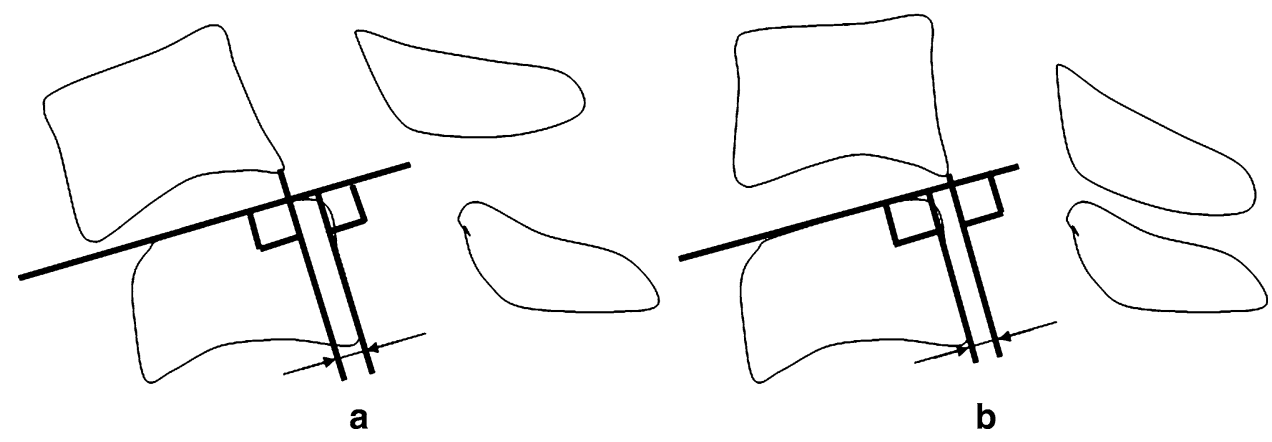

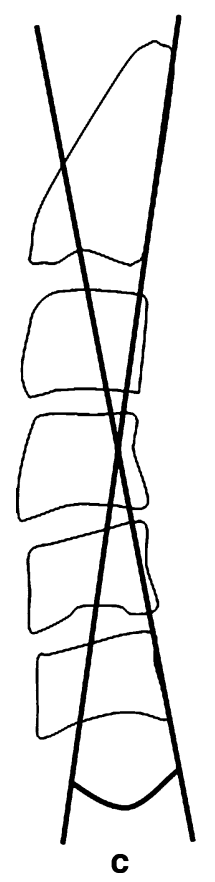

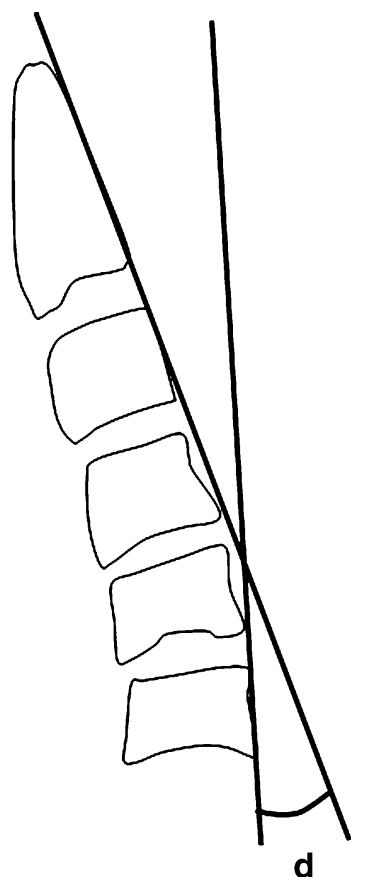

Fig. 3 Measurement of range of motion (ROM) of the cervical spine using functional radiographs. $\mathrm{ROM}=\mathrm{c}+\mathrm{d}$

between the DS group and the non-DS group $(42.4 \pm 21.2$ vs. $50.3 \pm 18.9 \%$, respectively; $p=0.27$; Table 2 ).

\section{Radiological findings}

There was a significant difference in the mean maximum slippage displacement between the DS group and the nonDS group $(3.1 \pm 0.6$ vs. $0.3 \pm 0.4 \mathrm{~mm}$, respectively; $p<0.01$; Table 1). The 15 patients in the DS group had 22 slippages of $2.5 \mathrm{~mm}$ or more. Of the 22 slippages, 3 were at $\mathrm{C} 2$ on $\mathrm{C} 3,9$ at $\mathrm{C} 3$ on $\mathrm{C} 4,9$ at $\mathrm{C} 4$ on $\mathrm{C} 5$, and 1 at $\mathrm{C} 5$ on $\mathrm{C} 6$ (Fig. 4).

With respect to the preoperative sagittal alignment of the cervical spine, in the DS group, lordotic, straight and kyphotic spines were found in 11,3 and 1 patient, respectively, while in the non-DS group, lordotic, straight and kyphotic spines were found in 14, 2 and 1 patient,
Table 1 Demographics of patients

\begin{tabular}{lllr}
\hline Group & DS group & $\begin{array}{l}\text { Non-DS } \\
\text { group }\end{array}$ & $p$ value \\
\hline $\begin{array}{l}\text { Age (years) } \\
\text { Sex }\end{array}$ & $72.4 \pm 6.3$ & $72.8 \pm 6.6$ & 0.87 \\
$\quad$ Male & 9 & 14 & \\
$\quad$ Female & 6 & 3 & $0.29^{\mathrm{a}}$ \\
$\begin{array}{l}\text { Duration of symptoms } \\
\quad(\text { min) }\end{array}$ & $24.1 \pm 31.6$ & $29.3 \pm 76.3$ & 0.80 \\
$\begin{array}{l}\text { Preoperative JOA score } \\
\text { Lordotic angle }\left({ }^{\circ}\right)\end{array}$ & $6.5 \pm 3.4$ & $6.8 \pm 3.8$ & 0.82 \\
$\begin{array}{l}\text { ROM of cervical spine }\left(^{\circ}\right) \\
\text { Maximum slippage }\end{array}$ & $41.3 \pm 12.0 \pm 15.8$ & $16.8 \pm 10.5$ & 0.65 \\
$\quad$ distance (mm) & $3.1 \pm 0.6$ & $0.3 \pm 0.4$ & $<0.01$ \\
\hline a $\chi^{2}$ test & & & \\
\end{tabular}

Table 2 Postoperative data

\begin{tabular}{llll}
\hline & DS group & Non-DS group & $p$ value \\
\hline Lordotic angle $\left({ }^{\circ}\right)$ & $17.1 \pm 11.6$ & $13.8 \pm 17.5$ & 0.54 \\
ROM of cervical spine $\left({ }^{\circ}\right)$ & $29.6 \pm 12.0$ & $25.5 \pm 13.2$ & 0.37 \\
Rate of ROM reduction $(\%)^{\mathrm{a}}$ & $29.2 \pm 16.1$ & $19.9 \pm 46.6$ & 0.47 \\
JOA score & $10.9 \pm 3.3$ & $11.9 \pm 2.8$ & 0.35 \\
Recovery rate $(\%)$ & $42.4 \pm 21.2$ & $50.3 \pm 18.9$ & 0.27
\end{tabular}

${ }^{\text {a }}$ Rate of $\mathrm{ROM}$ reduction $=($ preoperative $\mathrm{ROM}-$ postoperative $\mathrm{ROM})$ /preoperative $\mathrm{ROM} \times 100(\%)$

respectively (Table 3). At 3 years after operation in the DS group, lordotic and straight spines were found in 12 and 3 patients, respectively; no kyphotic spines were found. At 3 years after operation in the non-DS group, lordotic, straight and kyphotic spines were found in eight, seven and two patients, respectively (Table 4). There were no significant differences in sagittal cervical alignments at either the preoperative stage or at 3 years after operation between the two groups $\left(\chi^{2}\right.$ analysis, $\mathrm{p}=0.16$ and 0.29 , respectively). There was no difference in the preoperative lordotic angle between the DS group and the non-DS group $\left(19.0 \pm 15.8\right.$ vs. $16.8 \pm 10.5^{\circ}$, respectively; $\left.p=0.65\right)$. At 3 years after operation, there was no difference in the 


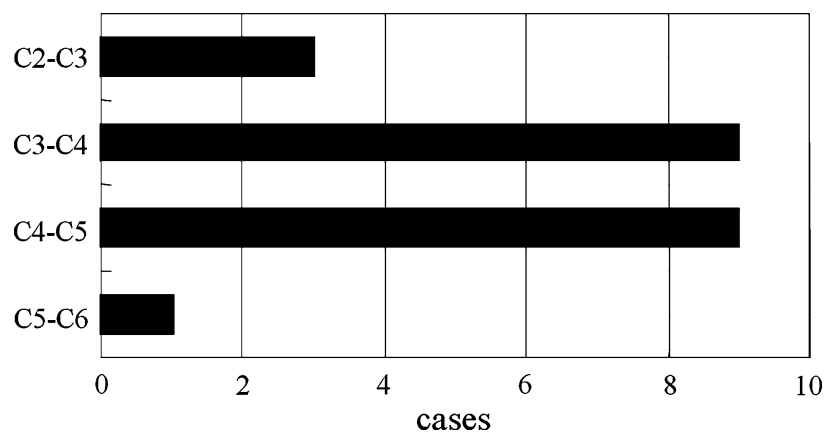

Fig. 4 Cases of slippage displacement that were $2.5 \mathrm{~mm}$ or more at each level

Table 3 Alignment of cervical spine preoperatively

\begin{tabular}{lllll}
\hline & Lordosis & Straight & Kyphosis & All \\
\hline DS group & 11 & 3 & 1 & 15 \\
Non-DS group & 14 & 2 & 1 & 17 \\
\hline
\end{tabular}

$p=0.16$

Table 4 Alignment of cervical spine postoperation

\begin{tabular}{lllll}
\hline & Lordosis & Straight & Kyphosis & All \\
\hline DS group & 12 & 3 & 0 & 15 \\
Non-DS group & 8 & 7 & 2 & 17 \\
\hline
\end{tabular}

$p=0.29$

lordotic angle between the DS group and the non-DS group $\left(17.1 \pm 11.6\right.$ vs. $13.8 \pm 17.5^{\circ}$, respectively; $\left.p=0.54\right)$ (Tables 1 and 2).

The range of motion of all cervical spines (DS group and non-DS group) was significantly limited from $37.7 \pm 13.4^{\circ}$ preoperatively to $27.4 \pm 12.6^{\circ}$ at 3 years after operation $(p<0.01)$. There was no significant difference in the range of motion of the cervical spine preoperatively and at 3 years after operation between the DS group and the nonDS group (Tables 1 and 2).

Postoperative spondylolisthesis

Spondylolisthesis disappeared postoperatively in 12 of 15 preoperative DS cases and decreased in 1 case. A total of $86.7 \%$ of DS cases had stabilized. Newly developed spondylolisthesis was detected in two cases in the DS group, while two non-DS cases changed to DS after 3 years (2/17 cases; $11.8 \%)$. A total of four cases had newly postoperative spondylolisthesis, with a recovery rate of $43.4 \pm 24.1(18-75 \%)$. These recovery rates were comparable to the total patient recovery rate.
Table 5 Changes in slippage displacement

\begin{tabular}{llll}
\hline & Preoperation & Postoperation & $p$ value \\
\hline C2-C3 & $1.2 \pm 1.5$ & $0.6 \pm 0.8$ & 0.21 \\
C3-C4 & $1.9 \pm 1.3$ & $0.8 \pm 1.0$ & 0.01 \\
C4-C5 & $1.8 \pm 1.3$ & $0.8 \pm 0.8$ & 0.02 \\
C5-C6 & $0.6 \pm 0.8$ & $0.7 \pm 1.2$ & 0.93 \\
\hline
\end{tabular}

\section{Discussion}

The results from the present study suggest that DS of the cervical spine is relatively common in elderly CSM patients [10], with a DS of $2.5 \mathrm{~mm}$ or more occurring in $30.6 \%$ of cases. Furthermore, DS seemed to have a predilection for the upper cervical levels among elderly patients, with $81.8 \%$ of the slippage displacements involving the C3-C4 or C4-C5 levels (Table 5). However, the majority of DS cases had stabilized after laminoplasty (86.7\%).

Spondylolisthesis is rarely observed in the cervical spine and has not been widely studied. However, more recent studies have focused on the DS of the cervical spine [2, 4, $28,30]$. There are a high number of patients with CSM in Japan [16], potentially due to the developmental spinal canal size [31], which was shown to be $2.25 \mathrm{~mm}$ smaller in Japanese than in Caucasian adults [19]. We also previously demonstrated that developmental canal stenosis occurred in $50.9 \%$ of CSM patients who were operated in our hospital [24]. These data suggest that most CSM patients frequently have more than two or three levels of cord compression, and we frequently perform laminoplasty in those patients. However, DS is considered to be an instability factor, and arthrodesis is considered the appropriate treatment for instability $[2,4,22,30]$.

In the present study, we retrospectively reviewed the cases of laminoplasty in elderly patients in our hospital and found that DS did not influence neurological recovery, with no difference in the preoperative and postoperative JOA scores or in the recovery rate between the DS and non-DS groups. This may be due to the change of mobility of the cervical spine after laminoplasty, as in several reports concerning the mobility of the cervical spine after laminoplasty, the postoperative range of motion was limited to approximately $50-60 \%$ of the preoperative range $[15,20,27]$. Similarly, in the present study, the range of motion of all cases was limited to $75.7 \%$ of the preoperative range, while slippage displacement was significantly restricted (slippage displacement in the DS group significantly decreased from $1.9 \pm 1.3$ to $0.8 \pm 1.0 \mathrm{~mm}$ at $\mathrm{C} 3-\mathrm{C} 4$ and $1.8 \pm 1.3$ to $0.8 \pm 0.8 \mathrm{~mm}$ at $\mathrm{C} 4-\mathrm{C} 5)$. It was also previously reported that $85.2 \%$ of spondylolisthesis cases stabilized slippage after laminoplasty [23]. We suggest that these restrictions result from facet joint contracture secondary to the creation 
of lateral gutters and/or from soft tissue contracture after laminoplasty. Thus, it is likely that immobilization is beneficial for cases of CSM with DS.

We observed a high incidence of DS at the upper cervical level (C3-C4 or C4-C5) in elderly patients with CSM. In a previous study comparing the dynamic radiological characteristics of 100 normal subjects older than 60 years with those of younger subjects, narrowing of the discs and osteophytosis were common in the older group at the C5-C6 and C6-C7 levels, with a significant decrease in intervertebral mobility and a comparatively greater mobility at the higher C3-C4 and C4-C5 levels [9]. The lower and middle levels of the cervical spine have also been shown to exhibit different patterns of movement [21], with some sliding of the superior vertebrae over the inferior vertebrae at $\mathrm{C} 3-\mathrm{C} 4$ and $\mathrm{C} 4-\mathrm{C} 5$, while little sliding at the C5-C6 and C6-C7 levels. Thus, the preferential location of DS at C3-C4 and C4-C5 may be explained by the relative hypermobility and the different pattern of movement.

In this study, we included kyphosis cases (DS: one case, $5^{\circ}$ kyphosis; and non-DS: one case, $9^{\circ}$ kypnosis). Generally, kyphosis might have always been taught as a contraindication for laminoplasty decompression in CSM. However, we consider that kyphosis cases are not always contraindications. Suda $\mathrm{K}$ et al. analyzed the operative indication with respect to cervical alignment using logistic regression analysis [25]. In that study, the highest risk of poor recovery was local kyphosis exceeding $13^{\circ}$ for CSM in the presence of local kyphosis. When patients have local kyphosis exceeding $13^{\circ}$, anterior decompression or posterior correction of kyphosis as well as laminoplasty should be considered. Laminoplasty consists of two distinct mechanisms for its decompression effect: a direct posterior decompression effect and an indirect anterior decompression effect resulting from the posterior shift of the spinal cord from the anterior compressive lesions. Our kyphosis cases may be insufficient for the posterior shift. However, we think that there was a direct posterior decompression effect. Therefore, we think that mild slippage with 'small kyphosis' is tolerable, but not when the kyphosis is big, exceeding $13^{\circ}$.

There are several limitations in the present study. First, we only evaluated radiographs and could not include preoperative MRI data, without which it is difficult to assess soft tissue compressive elements. Therefore, we could not evaluate the precise correlation between the instability and stenosis. Moreover, there were no recorded magnification factors in the previously taken radiographs. Magnification depends on the distance from the film to the cervical spine. This distance may vary for each case, and it is difficult to record magnification for all cases. Second, we only reviewed patients who received laminoplasty in our hospital. We have not yet investigated DS among asymptomatic healthy people and could not determine the frequency of DS in asymptomatic elderly people. Third, we were unable to evaluate the maximum tolerable slippage due to the retrospective design of our study. This will form the basis for future work.

\section{Conclusions}

We evaluated the clinical significance of preoperative DS for patients with CSM treated with bilateral open-door laminoplasty. DS was observed in $30.6 \%$ of our elderly CSM patients. Moreover, DS seemed to have a predilection for the upper cervical levels. However, there was no difference in the surgical results between the two groups, although we believe that DS is an important factor influencing CSM. Furthermore, DS did not affect improvement of neurological function. Laminoplasty without fusion can achieve good neurological improvement for CSM patients with DS.

\section{References}

1. al-Mefty O, Harkey HL, Marawi I, Haines DE, Peeler DF, Wilner HI, Smith RR, Holaday HR, Haining JL, Russell WF (1993) Experimental chronic compressive myelopathy. J Neurosurg 79:550-561

2. Boulos AS, Lovely TJ (1996) Degenerative cervical spondylolisthesis: diagnosis and management in five cases. J Spinal Disord 9:241-245

3. Dawley JA (1971) Spondylolisthesis of the cervical spine: case report. J Neurosurg 34:99-101

4. Deburg A, Mazda K, Guigui P (1995) Unstable degenerative spondylolisthesis of the cervical spine. J Bone Joint Surg Br $77: 122-125$

5. Durbin FC (1956) Spondylolisthesis of the cervical spine. J Bone Joint Surg Br 38:734-735

6. Ebersold MJ, Pare MC, Quast LM (1995) Surgical treatment for cervical spondylotic myelopathy. J Neurosurg 82:745-751

7. Fukui K, Kataoka O, Sho T, Sumi M (1990) Pathomechanism, pathogenesis, and results of treatment in cervical spondylotic myelopathy caused by dynamic canal stenosis. Spine 15:11481152

8. Harkey HL, Al-Mefty O, Marawi I, Peeler DF, Haines DE, Alexander LF (1995) Experimental chronic compressive myelopathy: effects of decompression. J Neurosurg 83:336-341

9. Hayashi H, Okada K, Hamada M, Tada K, Ueno R (1987) Etiologic factors of myelopathy: a radiographic evaluation of the ageing changes in the cervical spine. Clin Orthop 214:200-209

10. Hayashi H, Okada K, Hashimoto J, Tada K, Ueno R (1988) Cervical spondylotic myelopathy in the aged patient: a radiographic evaluation of the aging changes in the cervical spine and etiologic factors of myelopathy. Spine 13:618-625

11. Hirabayashi K, Miyakawa J, Satomi K, Maruyama T, Wakano K (1981) Operative results and postoperative progression of ossification among patients with ossification of cervical posterior longitudinal ligament. Spine 6:354-364

12. Japanese Orthopedic Association. Scoring system for cervical myelopathy. (1994) J Jpn Ortop Assoc 68: 490-503 
13. Kawakami M, Tamaki T, Iwasaki H, Yoshida $M$, Ando $M$, Yamada H (2000) A comparative study of surgical approaches for cervical compressive myelopathy. Clin Orthop 381:129-136

14. Kawasaki M, Tani T, Ushida T, Ishida K (2007) Anterolisthesis and retrolisthesis of the cervical spine in cervical spondylotic myelopathy in the elderly. J Ortho Sci 12:207-213

15. Kikuchi Y, Baba H, Tomita K, Umeda S (1991) Radiographic studies of the cervical spine after expansive laminoplasty. Seikei Saigaigeka 34:165-170 (Japanese)

16. Kokubun S, Sato T, Ishii Y, Tanaka Y (1996) Cervical myelopathy in Japan. Clin Orthop Relat Res 323:129-138

17. Kosnik EJ, Johnson JC, Scoles PV, Rossel CW (1979) Cervical spondylolisthesis. Spine 4:203-205

18. Matsuzaki H, Hoshino M, Kiuchi T, Toriyama S (1989) Domelike expansive laminoplasty for the second cervical vertebra. Spine 14:1198-1203

19. Murone I (1974) The importance of the sagittal diameters of the cervical spinal canal in relation to spondylosis and myelopathy. J Bone Joint Surg Br 56:30-36

20. Omura F, Shirai Y, Nakagawa S, Yamaguchi J, Fujiwara A, Ooba S (1989) Cervical spine motion after expansive laminoplasty: open-door laminoplasty vs split spinous process method. Rinsho Seikei Geka 24:453-459 (Japanese)

21. Penning L (1978) Normal movements of the cervical spine. AJR Am J Roentogenol 130:317-326

22. Pierre G, Michel B, Alain D (1998) Spinal deformity and instability after multilevel cervical laminectomy for spondylotic myelopathy. Spine 23:440-447

23. Sakai Y, Matsuyama Y, Inoue K, Ishiguro N (2005) Postoperative instability after laminoplasty for cervical myelopathy with spondylolisthesis. J Spinal Disord Tech 18:1-5
24. Shigematsu H, Ueda Y, Koizumi M, Takeshima T, Tanaka Y, Satoh N, Matsumori H, Oshima T, Matsuyama E, Kugai A, Takakura Y (2008) Does developmental canal stenosis influence surgical results of bilateral open-door laminoplasty for cervical spondylotic myelopathy? J Neurosurg Spine 9:358-362

25. Suda K, Abumi K, Ito M, Shono Y, Kaneda K, Fujiya M (2003) Local kyphosis reduces surgical outcomes of expansive opendoor laminoplasty for cervical spondylotic myelopathy. Spine 28:1258-1262

26. Sumi M, Kataoka O, Sawamura S, Ikeda M, Mukai H (1998) Radiological analysis of cervical spondylotic myelopathy. Rinsho Seikeigeka 33:1277-1286 (Japanese)

27. Sunago K, Kawai S, Oda Y (1989) Postoperative radiographic evaluation of cervical laminoplasty (Hattori's method). Rinsho Seikeigeka 24:445-452 (Japanese)

28. Tani T, Kawasaki M, Taniguchi S, Ushida T (2003) Functional importance of degenerative spondylolisthesis in cervical spondylotic myelopathy in the elderly. Spine 28:1128-1134

29. White AA, Panjabi MM (1988) Biomechanical considerations in the surgical management of cervical spondylotic myelopathy. Spine 13:15-20

30. Woiciechowsky C, Thomale U, Kroppenstedt S (2004) Degenerative spondylolisthesis of cervical spine-symptoms and surgical strategies depending on disease progress. Eur Spine J 13:680-684

31. Yonenobu K (2000) Cervical radiculopathy and myelopathy: when and what can surgery contribute to treatment? Eur Spine J 9:1-7 